\title{
Why heat transfer coefficients are unnecessary and undesirable, and how heat transfer problems are solved without them.
}

\section{EUGENE ADIUTORI ( $\sim$ EFADIUTORI@aol.com )}

\section{Original Article}

Keywords: dimensional homogeneity, heat transfer coefficient, law of convection heat transfer, nonlinear problems, parameter symbols

Posted Date: July 16th, 2020

DOI: https://doi.org/10.21203/rs.3.rs-41275/v1

License: (c) (1) This work is licensed under a Creative Commons Attribution 4.0 International License. Read Full License 


\title{
Why heat transfer coefficients are unnecessary and undesirable, and how heat transfer problems are solved without them.
}

\author{
Eugene F. Adiutori \\ Ventuno Press \\ 1094 Sixth Lane N. \\ Naples, FL 34102 \\ efadiutori@aol.com \\ $1-239-537-4107$ \\ July 8,2020
}

\begin{abstract}
Heat transfer coefficients (h) are unnecessary and undesirable. They are unnecessary because heat transfer problems are readily solved without them. They are undesirable because they greatly complicate problems that concern nonlinear thermal behavior.

In order to understand why heat transfer coefficients are unnecessary and undesirable, it is necessary to know precisely what $h$ is. The nomenclature in every heat transfer text should state " $h$ is a symbol for $q / \Delta T$ ". (Note that $q=h \Delta T$ and $h=q / \Delta T$ are identical.)

Problems in convection heat transfer are conventionally solved using $q, h$, and $\Delta T$-ie using $q$, $q / \Delta T$, and $\Delta T$. It is self-evident that any problem that can be solved using $q, q / \Delta T$, and $\Delta T$ can also be solved using only $q$ and $\Delta T$. Therefore $h$ (ie $q / \Delta T$ ) is unnecessary.

$h($ ie $q / \Delta T)$ is undesirable because, when $\mathrm{q}$ is a nonlinear function of $\Delta \mathrm{T}$ (as in free convection, condensation, and boiling), $h$ (ie $q / \Delta T$ ) is a third variable, and it greatly complicates problem solutions.

The text includes example problems that support the conclusion that $h$ (ie $q / \Delta T$ ) is unnecessary and undesirable.

Keywords: dimensional homogeneity; heat transfer coefficient; law of convection heat transfer; nonlinear problems; parameter symbols.
\end{abstract}


1. The genesis of $h$ and Eq. (1).

American heat transfer texts generally refer to Eq. (1) as "Newton's law of cooling", and credit Newton (1701) with $h$ and Eq. (1).

$q=h \Delta T$

However, Adiutori (1990) and Bejan (2013) state that Fourier (1822) should be credited with $h$ and Eq. (1).

Equation (1) cannot be "Newton's law of cooling" because cooling is a transient phenomenon, and Eq. (1) is a steady-state equation.

Newton could not have conceived $h$ or Eq. (1) because, in the eighteenth century, Eq. (1) was irrational. Until the nineteenth century, the science community globally agreed that parameters cannot rationally be multiplied, and Eq. (1) requires that parameters $h$ and $\Delta T$ be multiplied. Note that Newton's (1726) second law of motion presented in The Principia is not Eq. (2) because, to Newton and his colleagues, Eq. (2) was irrational. Newton's version of his second law of motion, as presented in The Principia, is Proportion (3).

$f=m a$

$a \alpha f$

\section{What exactly is $h$ ?}

Equations (1) and (4) are identical. Both equations state that $h$ is a symbol for $q / \Delta T$.

$q=h \Delta T$

$h=q / \Delta T$

The nomenclature in every heat transfer text should state " $h$ is a symbol for $q / \Delta T$ ".

\section{Fourier's view of Eq. (1) and $h$.}

Fourier (1822) performed experiments on steady-state forced convection heat transfer to ambient air. He conceived $h$ and Eq. (1) to correlate his data. In Fourier's view:

- Equation (1) states that, if heat transfer is by steady-state forced convection to ambient air, $q$ is always proportional to $\Delta T$.

- Equation (1) states that, if heat transfer is by steady-state forced convection to ambient air, $h$ is always a constant of proportionality.

- Equation (1) is the law of steady-state forced convection heat transfer to ambient air. It is a law because it is always obeyed. 


\section{The modern Eq. (1) and $h$.}

Sometime near the beginning of the twentieth century, it was decided to apply Eq. (1) to all forms of convection heat transfer. This decision had the following effects:

- Equation (1) ceased to apply only to proportional behavior, and began to also apply to linear and nonlinear behavior.

- h ceased to be a constant of proportionality, and became either a constant of proportionality or a variable.

\section{Why the modern Eq. (1) is neither an equation nor a law. It is a definition.}

It is self-evident that a proportional equation cannot describe linear or nonlinear behavior. If a proportional equation is applied to linear or nonlinear behavior, it ceases to be an equation, and becomes a definition of the coefficient in the proportional equation.

When it was decided to apply Eq. (1) to linear and nonlinear behavior, Eq. (1) ceased to be an equation, and became a definition of $h$. The modern Eq. (1) defines $h$ to be a symbol for $q / \Delta T-$ ie it states that $h$ and $q / \Delta T$ are identical.

Because the modern Eq. (1) is not an equation, it cannot be a law. There has in fact been no law of convective heat transfer since Eq. (1) ceased to be a law.

\section{Why $h$ (ie $q / \Delta T$ ) is unnecessary.}

$h$ (ie $q / \Delta T$ ) is unnecessary because, if a problem can be solved using $q, q / \Delta T$ (ie $h$ ), and $\Delta T$, it can also be solved using only $q$ and $\Delta T$.

\section{Why $h$ (ie $q / \Delta T$ ) is undesirable.}

If the relationship between $q$ and $\Delta T$ is proportional (as in forced convection to one phase fluid), $h$ (ie $q / \Delta T$ ) is a constant, and Eq. (1) contains the two variables $q$ and $\Delta T$. But if the relationship is nonlinear (as in free convection, condensation, and boiling), $h$ (ie $q / \Delta T$ ) is a variable, and Eq. (1) contains the three variables $q, h$ (ie $q / \Delta T$ ), and $\Delta T$.

$h$ (ie $q / \Delta T$ ) is undesirable because, if the relationship between $q$ and $\Delta T$ is nonlinear (as in free convection, condensation, and boiling), $h$ (ie $q / \Delta T$ ) is a third variable, and it greatly complicates solutions.

\section{How to eliminate $h$ (ie $q / \Delta T$ ) from heat transfer texts.}

In order to eliminate $h$ from heat transfer texts, substitute $q / \Delta T$ for $h$ and for $k / t$ in all equations that implicitly or explicitly include $h$, then separate $q$ and $\Delta T$.

For example, Eq. (5) is used to analyze heat transfer between two fluids separated by a flat wall.

$$
U=\left(1 / h_{1}+t_{\text {wall }} / k_{\text {wall }}+1 / h_{2}\right)^{-1}
$$


To separate $q$ and $\Delta T$ in Eq. (5):

- Substitute $q / \Delta T_{\text {total }}$ for $U$. Substitute $q / \Delta T_{1}$ for $h_{1}$ and $q / \Delta T_{2}$ for $h_{2}$.

- Substitute $q / \Delta T_{\text {wall }}$ for $k_{\text {wall }} / t_{\text {wall }}$.

- Separate $q$ and $\Delta T$, resulting in Eq. (6).

$\Delta T_{\text {total }}=\Delta T_{1}\{q\}+\Delta T_{\text {wall }}\{q\}+\Delta T_{2}\{q\}$

Equations (5) and (6) are identical - they differ only in form. Therefore, any problem that can be solved using Eq. (5) and $h$ can also be solved using Eq. (6) and not $h$.

Equation (7) is a heat transfer coefficient correlation often used in the analysis of forced convection heat transfer. To separate $q$ and $\Delta T$ in Eq. (7), replace Nu with $q D / \Delta T k$, then separate $q$ and $\Delta T$, resulting in Eq. (8a) or (8b).

$\mathrm{Nu} \equiv q D / \Delta T k=b \operatorname{Re}^{\mathrm{c}} \mathrm{Pr}^{\mathrm{d}}$

$q\{\Delta T\}=b(\Delta T k / D) \operatorname{Re}^{\mathrm{c}} \mathrm{Pr}^{\mathrm{d}}$

$\Delta T\{q\}=(q D / k)\left(b \operatorname{Re}^{\mathrm{c}} \operatorname{Pr}^{\mathrm{d}}\right)^{-1}$

9 Without using $h$, solve a heat transfer problem in which the relationship between $q$ and $\Delta T$ is proportional (as in forced convection heat transfer).

9.1 Problem 1, Statement

Without using $h$, calculate the heat flux through a flat wall that separates Fluids 1 and 2.

\subsection{Problem 1, Given}

Parameter dimension units are specified in Nomenclature.

$T_{1}=440$

$T_{2}=85$

$\Delta T_{l}=.05 q \quad$ (identical to $\left.h_{l}=20\right)$

$\Delta T_{w}=.01 q \quad$ (identical to $\left.k_{\text {wall }} / t_{\text {wall }}=100\right)$

$\Delta T_{2}=.025 q \quad$ (identical to $\left.h_{2}=40\right)$

$\Delta T_{\text {total }}=\Delta T_{1\{}\{q\}+\Delta T_{\text {wall }}\{q\}+\Delta T_{2}\{q\} \quad$ (Identical to Eq. (5)) 


\subsection{Problem 1, Analysis and solution}

Combine Eqs. (11 to (14), resulting in Eq. (15).

$(440-85)=.05 q+.01 q+.025 q=.085 q$

$q=355 / .085=4,180$

\subsection{Problem 1, Conclusions}

Problem 1 is simple to solve whether $h$ is or is not used in the solution because $q / \Delta T$ is a constant. Consequently the problem solution contains only two thermal variables whether $h$ is or is not used in the solution.

10. Without using $h$, solve a heat transfer problem in which the relationship between $q$ and $\Delta T$ is moderately nonlinear (as in free convection heat transfer).

\subsection{Problem 2, Statement}

Calculate the heat flux through a flat wall that separates Fluids 1 and 2.

\subsection{Problem 2, Given}

Parameter dimension units are specified in Nomenclature.

$T_{1}=440$

$T_{2}=85$

$\Delta T_{1}=.0910 q^{0.80} \quad$ (identical to $h_{1}=20 \Delta T_{1}{ }^{.25}$ )

$\Delta T_{\text {wall }}=.010 q \quad$ (identical to $\left.k_{\text {wall }} / t_{\text {wall }}=100\right)$

$\Delta T_{2}=.0523 q^{0.80} \quad$ (identical to $h_{2}=40 \Delta T_{2}{ }^{.25}$ )

\subsection{Analysis and solution, Problem 2}

$\Delta T_{\text {total }}=\Delta T_{1}\{q\}+\Delta T_{\text {wall }}\{q\}+\Delta T_{2}\{q\} \quad$ (identical to Eq. (5))

$(440-85)=.0910 q^{0.80}+.010 q+.0523 q^{0.80}$

$q=11,000$

10.4 Analysis of Problem 2 using $h$ methodology

If $h$ methodology is used, the analysis is based on Eq. (25), and results in Eq. (26).

$U=\left(1 / h_{1}+t_{\text {wall }} / k_{\text {wall }}+1 / h_{2}\right)^{-1}$

$U=\left(1 / 20 \Delta T_{1}^{.25}+1 / 100+1 / 40 \Delta T_{2}^{.25}\right)^{-1}$ 


\subsection{Problem 2 Conclusions}

Problem 2 demonstrates that moderately nonlinear problems are much more difficult to solve if $h$ is used in the solution. Note that Eq. (23) has only one unknown variable, and can be solved by anyone in about a minute using Excel and trial-and-error methodology. Also note that Eq. (26) has three unknown variables $\left(U, \Delta T_{1}\right.$, and $\left.\Delta T_{2}\right)$, and can be solved only by someone who knows a good deal about heat transfer and mathematics. Also, Eq. (26) takes much longer than a minute to solve, and has a much greater likelihood of error. In order to solve Eq. (26), it is necessary to:

- Find two more equations that apply to the problem.

- Solve the three equations simultaneously to determine $q / \Delta T_{\text {total }}$-ie to determine $U$.

- Multiply $q / \Delta T_{\text {total }}$ times $\Delta T_{\text {total }}$ to determine $q$.

\section{Without using $h$, solve a heat transfer problem in which $q$ is a highly nonlinear function of $\Delta T$}

\subsection{Statement, Problem 3}

Given a vented pool boiler with a horizontal boiler plate, a boiling fluid above the boiler plate, and a heat source fluid below the boiler plate, determine the following without using $h$ :

- The heat flux through the boiler plate at various heat source temperatures.

- The thermal stability of the boiler at various heat source temperatures.

- The effect of thermal instability on boiler behavior.

\subsection{Given, Problem 3}

- Parameter dimension units are specified in Nomenclature.

- The heat transfer behavior of the boiling interface is qualitatively described in Figure 1. The $y$ axis is labeled $q_{o u t}$ because the chart concerns heat flux out of the boiling interface.

- The boiler heat source is Fluid 1 at temperature $T_{1}$.

- The boiling fluid is Fluid 2 at temperature $T_{\text {sat }} . T_{\text {sat }}$ is fixed because the boiler is vented.

- The behavior described in Figure 1 resembles the boiling behavior of liquid metals.

- The heat transfer in Region 1 of Figure 1 is by natural convection from the boiler plate to the surface of Fluid 2, and by evaporation at the surface.

- Region 2 is a dashed line because there is no steady-state solution in Region 2. However, the boiler behaves as though the dashed line accurately describes functionality in Region 2. 


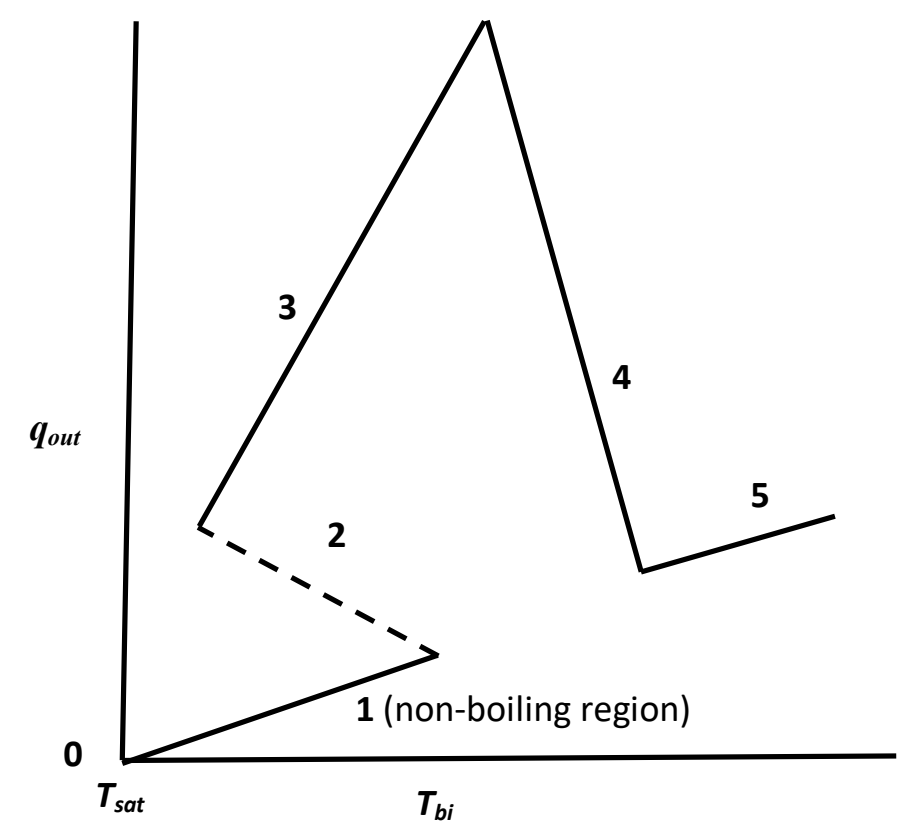

Figure 1 The thermal behavior of the boiling interface in Problem 3a

- The heat transfer behavior of the boundary layer on the Fluid 1 surface of the boiler plate is described by Eq. (27).

$\Delta T_{1}=b q$

- The heat transfer behavior of the boiler plate is described by Eq. (28).

$$
\Delta T_{b p}=c q
$$

\subsection{How to determine the boiler plate heat flux}

On Figure 1, plot $q_{i n}$ vs $\left(440-b q_{i n}-c q_{i n}\right) . q_{\text {in }}$ is the heat flux into the boiling interface. (440$\left.b q_{i n}-c q_{i n}\right)$ is the temperature of the boiling interface. The boiler plate heat flux is the heat flux at intersections of $q_{\text {in }}$ and $q_{\text {out }}$.

\subsection{How to appraise the thermal stability of the boiler}

The thermal stability of the boiler at an intersection of $q_{\text {in }}$ and $q_{\text {out }}$ is appraised by inspecting the intersection to determine whether a small perturbation would increase or decrease with time. If a small perturbation would decrease with time, the boiler is stable with regard to small perturbations. If a small perturbation would increase with time, the boiler is unstable. Symbolically, an intersection is thermally unstable if Inequality (29) is satisfied at the intersection.

$\mathrm{d} q_{i n} / \mathrm{d} T_{b i}>\mathrm{d} q_{o u t} / \mathrm{d} T_{b i}$ 
11.5 How to determine the boiler behavior that results from thermal instability

The boiler behavior that results from thermal instability is determined by inspecting intersections of $q_{\text {in }}$ and $q_{\text {out }}$, and adjacent regions. If an intersection is thermally unstable, inspection of the chart will indicate either hysteresis or undamped oscillation in boiler plate temperature and heat flux.

11.5.1 The thermal stability and boiler behavior that result if there is only one intersection of $q_{\text {in }}$ and $q_{o u t}$, and it is in Region 1, 3, 4, or 5 of Figure 1

If there is only one intersection of $q_{\text {in }}$ and $q_{\text {out }}$, and it is in Region 1, 3, 4, or 5 of Figure 1, inspection of Figure 1 indicates that a small perturbation at the intersection would decrease with time. Therefore all intersections indicate thermal stability with regard to small perturbations. However, a boiler that is thermally stable with regard to small perturbations may be thermally unstable with regard to large perturbations.

11.5.2 Thermal stability and boiler behavior if there is one intersection of $q_{\text {in }}$ and $q_{\text {out }}$, and it is in Region 2 of Figure 1

If there is only one intersection of $q_{i n}$ and $q_{\text {out }}$, and it is in Region 2 of Figure 1, inspection of the intersection and the adjacent regions indicates that small perturbations at the intersection increase with time, and result in undamped oscillations in heat flux and boiler plate temperature as operation jumps from the upper end of Region 1 to the upper part of Region 3, transitions to the lower end of Region 3, jumps down to the lower part of Region 1, transitions to the upper end of Region 1, jumps to the upper part of Region 3, etc.

11.5.3 Thermal stability and boiler behavior if there are three intersections of $q_{\text {in }}$ and $q_{\text {out }}$, and they are in Regions 1, 2, and 3 of Fig. 1

If there are three intersections of $q_{i n}$ and $q_{\text {out }}$, and they are in Regions 1, 2, and 3 of Figure 1, inspection of the intersections and adjacent regions indicates that:

- The boiler operates in a stable manner in either Region 1 or Region 3.

- There are step changes in $q_{o u t}$ as $T_{b i}$ is monotonically increased from Region 1 to Region 3, and as $T_{b i}$ is monotonically decreased from Region 3 to Region 1.

- There are no undamped oscillations because, if the boiler is initially at an intersection in Region 2, it will transition to an intersection in either Region 1 or Region 3 where it will operate in a stable manner.

11.6 Solution of Problem 3 using $h$ methodology.

It would be extremely difficult to solve Problem 3 using $h$ methodology, as evidenced by the fact that an $h$ methodology solution of a problem like Problem 3 is not found in American heat transfer texts or journals.

\subsection{Conclusions based on Problems 1 to 3}

- Problem 1 demonstrates that proportional problems are easily solved whether $h$ is or is not used in the solution. 
- Problem 2 demonstrates that moderately nonlinear problems are much simpler to solve if $h$ is not used in the solution.

- Problem 3 demonstrates that highly nonlinear problems are simple to solve if $h$ methodology is not used in the solution. It would be extremely difficult to solve Problem 3 using $h$ methodology, as evidenced by the fact that an $h$ methodology solution of a problem like Problem 3 is not found in American heat transfer texts or journals.

\section{Conclusions}

Heat transfer coefficients are unnecessary and undesirable, and should be abandoned.

\section{Nomenclature}

\section{Symbols}

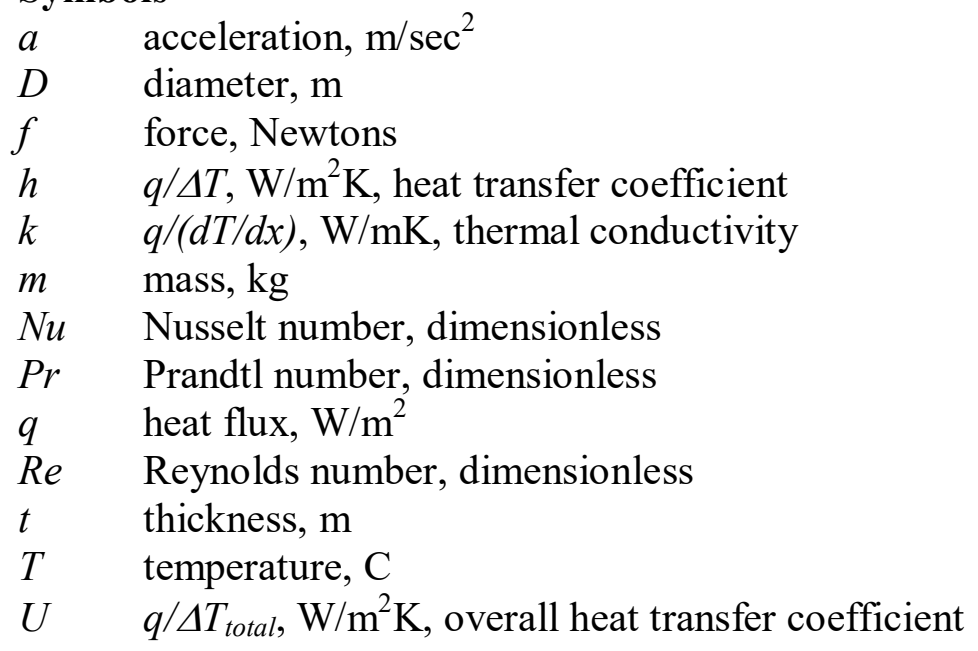

\section{Subscripts}

1 fluid number

2 fluid number

bi boiling interface

bp boiler plate

in into the interface

out out of the interface

sat saturated

\section{References}

Adiutori, E. F., 1990, "Origins of the Heat Transfer Coefficient", Mechanical Engineering, August, pp 46-50

Bejan, A., 2013, Convection Heat Transfer, $4^{\text {th }}$ edition, Wiley, p. 32 
Fourier, J., (1822), The Analytical Theory of Heat, 1955 Dover edition of the 1878 English translation, The University Press, Article 36

Newton, I., 1701, “A Scale of the Degrees of Heat”, Phil Trans Royal Soc (London), 22, p. 824

Newton, I., (1726), The Principia, 3rd edition, translation by Cohen, I. B. and Whitman, A. M., 1999, p. 460

Special section:

There are no data for this manuscript.

There are no competing interests.

There was no funding.

There is only one author.

There are no acknowledgements. 
Figures

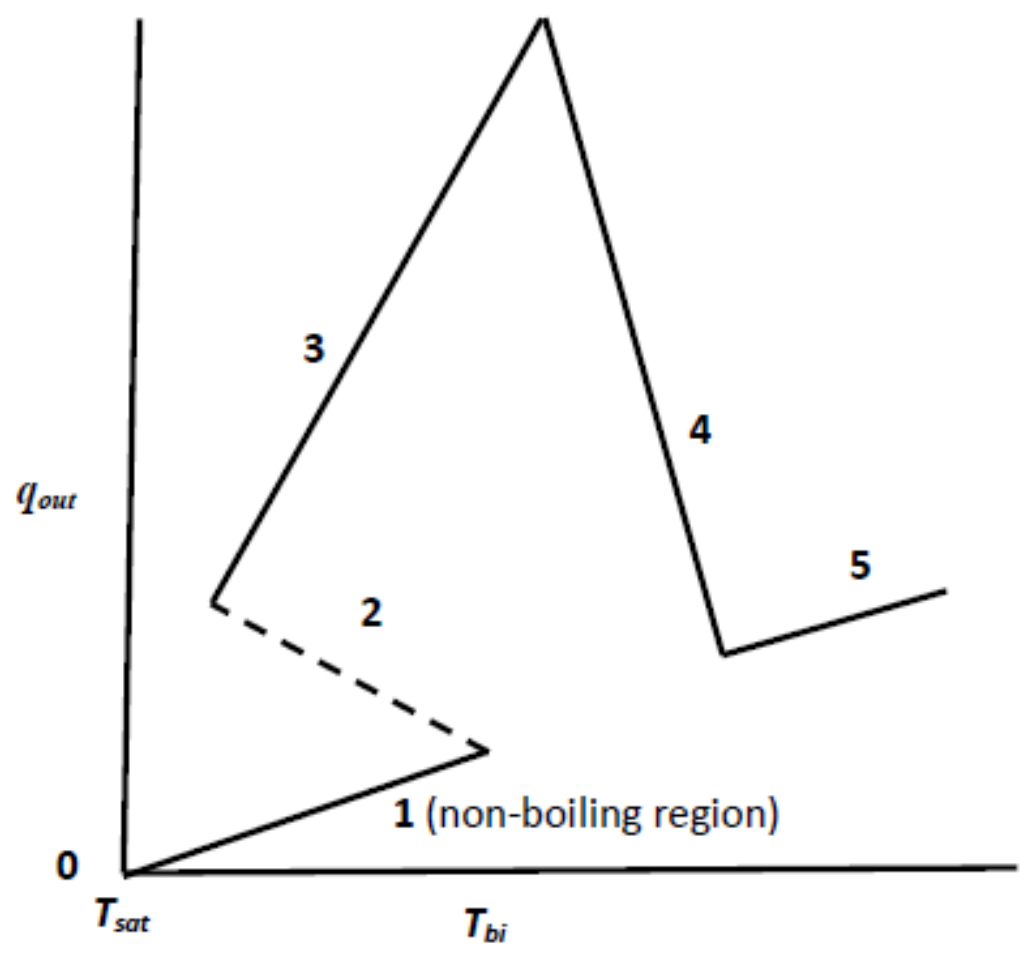

Figure 1

The thermal behavior of the boiling interface in Problem 3a 